\title{
Frequency modulation and pulse compression by coherent multimode molecular motion
}

\author{
Fam Le Kien ${ }^{\text {f }}$ and K. Hakuta \\ Department of Applied Physics and Chemistry, University of Electro-Communications, Chofu, Tokyo 182-8585, Japan \\ CREST, Japan Science and Technology Corporation (JST), Chofu, Tokyo 182-8585, Japan \\ A. V. Sokolov \\ Department of Physics and Institute for Quantum Studies, \\ Texas AEM University, College Station, TX 77843-4242
}

(Dated: November 20, 2018)

\begin{abstract}
We study beating of a probe field with a time-varying susceptibility in a coherently prepared Raman medium. We consider the general case of an arbitrary variation of susceptibility, which corresponds to a superposition of an arbitrary number of excited Raman transitions. We derive a general analytical solution and conservation relations for this process. We show that the interference between Raman polarizations may substantially affect frequency modulation and pulse compression for the probe field.

PACS numbers: 42.65.Re, 32.80.Qk, 42.50.Gy, 42.65.Sf
\end{abstract}

We have recently presented a detailed analysis of ultrashort laser pulse compression by parametric beating with a sinusoidal molecular oscillation [1]. In this Brief Report, we extend the previous work to the general case of an arbitrarily complex molecular motion. We assume a wave-like molecular excitation, produced by external fields in a dispersionless medium. Molecular motion results in a time-varying susceptibility, which (in the absence of dispersion) leads to time variations in phase and group velocities for a probe field [1]. We analyze the effect of these variations on pulse propagation, derive an analytical solution in terms of an integral for a time transformation, and use it to obtain conservation relations.

It was shown recently that coherently driven molecular oscillations can produce frequency modulation with ultrabroad bandwidth [2, 3], and result in subfemtosecond [4, 5, 6] and subcycle [7] pulse compression. A molecular oscillation can be either sinusoidal (a single coherently excited Raman transition) [2, 3, 4, 5, 6] or more complex (multimode vibrational or rotational molecular wave packets, which correspond to a coherent phased excitation of many Raman transitions) 8, 9]. Molecular wave packets can be excited either impulsively 6, 8, 9 , or by applying several quasi-monochromatic laser fields [7]. Generalization of our previous results [1] to the important case of a complex molecular motion will provide insight into the Raman pulse compression techniques.

The essence of our result is simple: A pulse is compressed or stretched by time-varying phase and group velocities, such that envelope compression or stretching is accompanied by frequency increase or decrease, respectively. Pulse amplitude and oscillation frequency are

*On leave from Department of Physics, University of Hanoi, Hanoi, Vietnam; also at Institute of Physics, National Center for Natural Sciences and Technology, Hanoi, Vietnam changed by reciprocal factors, such that the photon number, the pulse area, and the number of optical oscillations are conserved, while the pulse energy is not. When the resultant change in the pulse bandwidth is small compared to the optical carrier frequency, pulse deformation by the complex non-sinusoidal molecular motion can be obtained as a multiplication of the effects of individual harmonic components of the motion. However, for stronger modulation this intuitive thinking can not be applied. In order to emphasize this point, in the second part of our Report we consider an example of a biharmonic molecular excitation and show that frequency modulation and pulse compression are significantly affected by the interference between two Raman polarizations.

We study the propagation of a probe field $E$ in a Raman medium, characterized by a polarization $P$. In the local time $\tau=t-z / c$, the reduced wave equation reads

$$
\frac{\partial E}{\partial z}=-\frac{1}{2 \epsilon_{0} c} \frac{\partial P}{\partial \tau}
$$

We assume that dispersion is negligible. The instantaneous susceptibility is defined as $\chi=P / \epsilon_{0} E$. We assume that $\chi=\chi_{0}+\chi_{m}(\tau-z / v)$, where $\chi_{0}$ is the linear time-independent susceptibility, $\chi_{m}$ is the Raman timevarying susceptibility, and $v=2 c / \chi_{0}$ is the average phase and group velocity in the local time coordinates.

We use the reduced local time $\eta=\tau-z / v$. In the coordinates $z$ and $\eta$, Eq. (11) becomes

$$
\frac{\partial}{\partial z} E(z, \eta)=-\frac{1}{2 c} \frac{\partial}{\partial \eta} \chi_{m}(\eta) E(z, \eta)
$$

The solution to Eq. (2) is found to be

$$
E(z, \eta)=E_{\mathrm{in}}(s) G(\eta)
$$

where $E_{\text {in }}(s)=E(z=0, s)$ is the input field, $s$ is the 
input time determined from the output time $\eta$ by

$$
\int_{s}^{\eta} \frac{d \theta}{\chi_{m}(\theta)}=\frac{z}{2 c}
$$

and $G(\eta)$ is the compression factor given by

$$
G(\eta)=\frac{\chi_{m}(s)}{\chi_{m}(\eta)}
$$

Differentiating Eq. (4) with respect to $\eta$, we obtain

$$
\frac{d s}{d \eta}=G(\eta) .
$$

According to Eqs. (3) and (6), the height and duration of optical oscillations in the probe field are changed by the reciprocal factors $E(z, \eta) / E_{\text {in }}(s)=G(\eta)$ and $d \eta / d s=$ $1 / G(\eta)$, respectively. The value $G(\eta)>1(G(\eta)<1)$ indicates pulse compression (stretching) in the vicinity of $\eta$. From Eqs. (3) and (6), we find the relation

$$
\int_{\eta_{1}}^{\eta_{2}} E(z, \eta) d \eta=\int_{s_{1}}^{s_{2}} E_{\text {in }}(s) d s
$$

which describes the conservation of the pulse area.

We find from Eq. (6) that the instantaneous oscillation frequency of the output field is

$$
\omega_{\mathrm{osc}}(\eta)=G(\eta) \omega_{0},
$$

where $\omega_{0}$ is the input frequency. As seen, $\omega_{\text {osc }}(\eta)$ is modulated in time by the compression factor $G(\eta)$. Using Eqs. (3), (6), and (8), we find

$$
\frac{c \epsilon_{0}}{2} \int_{\eta_{1}}^{\eta_{2}} \frac{E^{2}(z, \eta)}{\hbar \omega_{\mathrm{osc}}(\eta)} d \eta=\frac{c \epsilon_{0}}{2} \int_{s_{1}}^{s_{2}} \frac{E_{\mathrm{in}}^{2}(s)}{\hbar \omega_{0}} d s .
$$

This relation describes the conservation of the photon number, which is always satisfied for Raman processes.

We introduce the mean frequency $\bar{\omega}\left(\eta_{1}, \eta_{2}\right)=\left(\eta_{2}-\right.$ $\left.\eta_{1}\right)^{-1} \int_{\eta_{1}}^{\eta_{2}} \omega_{\mathrm{osc}}(\eta) d \eta$ for the oscillations in the time interval $\left(\eta_{1}, \eta_{2}\right)$. Then, we find from Eqs. (6) and (8) that $\bar{\omega}\left(\eta_{1}, \eta_{2}\right)\left(\eta_{2}-\eta_{1}\right)=\omega_{0}\left(s_{2}-s_{1}\right)$, that is, the product of the pulse length and the mean frequency is constant during the propagation process. Furthermore, Eq. (3) says that $E(z, \eta)=0$ if $E_{\text {in }}(s)=0$ and vice versa, that is, a zero of the input field at an input time $s$ leads to a zero of the output field at the corresponding output time $\eta$. Hence, the number of optical oscillations is conserved.

To get insight into the behavior of the compression factor $G$, we derive an explicit, approximate expression for this factor. For this purpose, we consider a particular case where the deviation of an output time $\eta_{i}$ from its corresponding input time $s_{i}$ is small compared to the characteristic variation time $T_{m}$ of $\chi_{m}$, that is, $\left|s_{i}-\eta_{i}\right| \ll$ $T_{m}$. We take $\eta$ close to $\eta_{i}$, and use the approximation $\chi_{m}(\theta)=\chi_{m}\left(\eta_{i}\right)+\chi_{m}^{\prime}\left(\eta_{i}\right)\left(\theta-\eta_{i}\right)$ to calculate the integral in Eq. (画. Then, we find

$$
s-\eta_{i}+\frac{\chi_{m}\left(\eta_{i}\right)}{\chi_{m}^{\prime}\left(\eta_{i}\right)}=\left[\eta-\eta_{i}+\frac{\chi_{m}\left(\eta_{i}\right)}{\chi_{m}^{\prime}\left(\eta_{i}\right)}\right] \exp \left[-\frac{z}{2 c} \chi_{m}^{\prime}\left(\eta_{i}\right)\right] .
$$

Hence, Eq. (6) yields

$$
G\left(\eta_{i}\right)=\left.\frac{d s}{d \eta}\right|_{\eta=\eta_{i}}=\exp \left[-\frac{z}{2 c} \chi_{m}^{\prime}\left(\eta_{i}\right)\right] .
$$

Equation (11) shows that the factor $G$ is approximately determined by the time derivative of the Raman susceptibility $\chi_{m}$, multiplied with the propagation length $z$. Note that, around the chosen time $\eta_{i}$, the factor $G$ is multiplicative, that is, pulse deformation by the complex molecular motion can be obtained as a multiplication of the effects of individual components of the motion. The assumption $\left|s_{i}-\eta_{i}\right| \ll T_{m}$ requires

$$
\left|\frac{\exp \left[-(z / 2 c) \chi_{m}^{\prime}\left(\eta_{i}\right)\right]-1}{\chi_{m}^{\prime}\left(\eta_{i}\right)}\right| \ll \frac{T_{m}}{\left|\chi_{m}\left(\eta_{i}\right)\right|} .
$$

Condition (12) is satisfied when $(z / c)\left|\chi_{m}^{\prime}\left(\eta_{i}\right)\right| \ll 1$ and $(z / c)\left|\chi_{m}\left(\eta_{i}\right)\right| \ll T_{m}$, i.e., when the Raman susceptibility and its modulation are small. In this case, we have $G\left(\eta_{i}\right) \cong 1-(z / 2 c) \chi_{m}^{\prime}\left(\eta_{i}\right) \cong 1$, indicating that the pulse compression is weak. Condition (12) is also satisfied when $\eta_{i}$ is a zero of $\chi_{m}$.

In general, $G$ is not multiplicative with respect to the individual susceptibilities. To show this, we consider the case where the Raman susceptibility $\chi_{m}$ is a sum of two sinusoids corresponding to two individual Raman transitions, namely, $\chi_{m}=\chi_{a}+\chi_{b}$, where $\chi_{j}=\chi_{j}^{(0)} \sin \left(\omega_{j} \eta+\varphi_{j}\right)$ for $j=a, b$. The compression factor corresponding to the individual component $\chi_{j}$ is given by the periodic comb function [1, 10]

$$
G_{j}=\frac{1}{e^{\alpha_{j} z} \cos ^{2} \frac{\omega_{j} \eta+\varphi_{j}}{2}+e^{-\alpha_{j} z} \sin ^{2} \frac{\omega_{j} \eta+\varphi_{j}}{2}},
$$

where $\alpha_{j}=\left(\omega_{j} / 2 c\right) \chi_{j}^{(0)}$. In general, the total compression factor $G$ is different from the product of the individual compression factors $G_{a}$ and $G_{b}$. The reason is that the compression factor $G$ is produced by the timevarying Raman susceptibility $\chi_{m}$ via a nonlinear mechanism, see Eqs. (1) and (5). Due to the nonlinearity of this mechanism, the individual susceptibility components may interfere with each other in beating with the field.

To see the interference between the individual susceptibility components in beating with the field, we illustrate the functions $G$ and $G_{a} G_{b}$ in Figs. 11(a) and 1 (b), respectively. The plots are calculated for the parameters $\alpha_{a} z=0.8, \alpha_{b} z=0.6, \omega_{b}=0.07 \omega_{a}$, and $\varphi_{a}=\varphi_{b}=0$. The time is normalized to the Raman period $T_{a}=2 \pi / \omega_{a}$. Both $G$ and $G_{a} G_{b}$ reveal a sequence of teeth corresponding to the oscillation of the susceptibility component $\chi_{a}$, which has a higher frequency. The peaks of these teeth are modulated in accordance with the oscillation of the component $\chi_{b}$, which has a lower frequency. The differences between $G$ and $G_{a} G_{b}$ are clearly observed.

To see in detail the difference between $G$ and $G_{a} G_{b}$, we magnify in Fig. 2 the curves of Fig. 11 for the time windows $1 \leq \eta / T_{a} \leq 2$ (a) and $7.5 \leq \eta / T_{a} \leq 8.5$ (b). We 


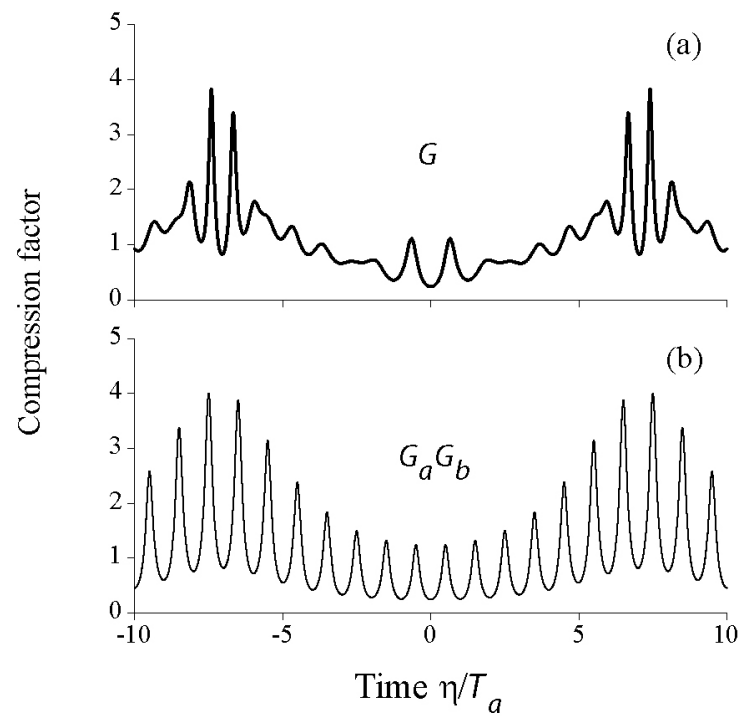

FIG. 1: Comparison between the total compression factor $G$ (a) and the product of individual compression factors $G_{a} G_{b}$ (b). The plots are calculated for the parameters $\alpha_{a} z=0.8$, $\alpha_{b} z=0.6, \omega_{b}=0.07 \omega_{a}$, and $\varphi_{a}=\varphi_{b}=0$.
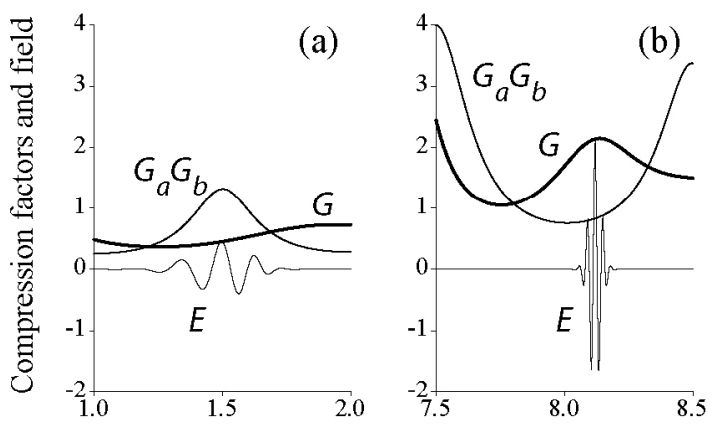

Time $\eta / T_{a}$

FIG. 2: Magnification of the curves for $G$ and $G_{a} G_{b}$ of Fig. 1 in the regions $1 \leq \eta / T_{a} \leq 2$ (a) and $7.5 \leq \eta / T_{a} \leq 8.5$ (b). The temporal profiles of a short probe field at the output in these time regions are also shown. The carrier frequency and pulse length of the input field are $\omega_{0}=15.2 \omega_{a}$ and $T=0.08 T_{a}$, respectively. The input peak time is $\eta_{p}=0.83 T_{a}$ (a) or $8.8 T_{a}$ (b). The peak value of the input field is one unit.

also plot in Fig. 2 the output field for the cases where the input peak time is $\eta_{p}=0.83 T_{a}$ (a) and $8.8 T_{a}(\mathrm{~b})$. The carrier frequency and pulse length of the input probe field are $\omega_{0}=15.2 \omega_{a}$ and $T=0.08 T_{a}$, respectively. We observe $G<1<G_{a} G_{b}$ and $G>1>G_{a} G_{b}$ around the times $\eta / T_{a}=1.5$ and 8.1, respectively. These relations imply that, due to the interference between $\chi_{a}$ and $\chi_{b}$, the total compression factor $G$ and the product of individual factors $G_{a} G_{b}$ may give opposite indications on pulse stretching and compression. Thus, the interference
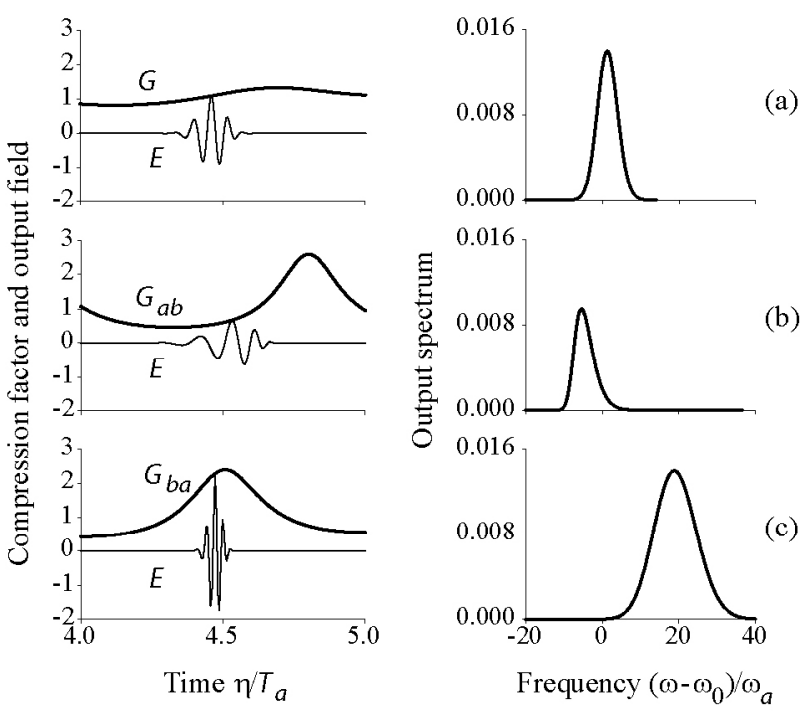

FIG. 3: Comparison between the mixture (a), the cascade $M_{a} M_{b}$ (b), and the cascade $M_{b} M_{a}$ (c) of the individual Raman systems $M_{a}$ and $M_{b}$. The parameters for the media are $\alpha_{a} z=\alpha_{a} z_{a}=0.8, \alpha_{b} z=\alpha_{b} z_{b}=0.6, \omega_{b}=0.07 \omega_{a}$, and $\varphi_{a}=\varphi_{b}=0$. The carrier frequency and pulse length of the input field are the same as for Fig. 2. The input peak time is $\eta_{p}=3.1 T_{a}$. The peak value of the input field is one unit.

between the susceptibility components can substantially affect frequency modulation and pulse compression.

In the above, the total compression factor $G$ has been compared with the product $G_{a} G_{b}$ of the individual compression factors. However, $G_{a} G_{b}$ cannot be rigorously considered as a compression factor for the case where there is no interference between $\chi_{a}$ and $\chi_{b}$. Below we will compare the compression factor of a combined system with the compression factor of a sequence of two individual systems.

We call $M_{a} M_{b}$ a cascade in which the probe field is sent through, first, a cell $M_{a}$ (with susceptibility $\chi_{a}$ and length $z_{a}$ ) and, then, a cell $M_{b}$ (with susceptibility $\chi_{b}$ and length $\left.z_{b}\right)$. For this configuration, we have $E_{\text {out }}(\eta)=$ $E_{\text {in }}(s) G_{a b}(\eta)$ and $d s / d \eta=G_{a b}(\eta)$, where

$$
G_{a b}(\eta)=G_{a}\left(\eta^{\prime}, z_{a}\right) G_{b}\left(\eta, z_{b}\right)
$$

Here $\eta^{\prime}$ and $s$ are determined by the equations 11,10

$$
\begin{gathered}
\tan \left[\left(\omega_{b} \eta^{\prime}+\varphi_{b}\right) / 2\right]=e^{-\alpha_{b} z_{b}} \tan \left[\left(\omega_{b} \eta+\varphi_{b}\right) / 2\right], \\
\tan \left[\left(\omega_{a} s+\varphi_{a}\right) / 2\right]=e^{-\alpha_{a} z_{a}} \tan \left[\left(\omega_{a} \eta^{\prime}+\varphi_{a}\right) / 2\right] .
\end{gathered}
$$

Note that $G_{a b}$ is not symmetric with respect to the indices $a$ and $b$, i.e., $G_{a b} \neq G_{b a}$. In other words, the cascades $M_{a} M_{b}$ and $M_{b} M_{a}$ produce different compression factors. This feature is different from the results of Ref. [7], which are valid for the case of limited bandwidths.

Comparison between Eqs. (5) and (14) shows that the compression factor $G$ of the combined system is different from the factors $G_{a b}$ and $G_{b a}$ of the cascades. To see 
the difference between the mixture and cascades of the individual systems $M_{a}$ and $M_{b}$, we plot the factors $G$, $G_{a b}$, and $G_{b a}$ in Figs. 3(a), 3(b), 3(c), respectively, for the time window $4 \leq \eta / T_{a} \leq 5$. We also plot the output field and the output spectrum for the input peak time $\eta_{p}=3.1 T_{a}$. The other parameters are the same as for Fig. 2. We observe $G_{a b}<G \cong 1<G_{b a}$ around the time $\eta / T_{a}=4.5$. These relations as well as the temporal and spectral profiles of the output field show clearly the differences between the combined and cascade systems in frequency modulation and pulse compression.

In the case where $\alpha_{j} z_{j} \ll 1$ for $j=a, b$, we find to lowest order in $\alpha_{j} z_{j}$ the compression factor $G_{a b}=1-$ $\sum_{j} \alpha_{j} z_{j} \cos \left(\omega_{j} \eta+\varphi_{j}\right)$ and the input-output time relation $s=\eta-\sum_{j}\left(\alpha_{j} z_{j} / \omega_{j}\right) \sin \left(\omega_{j} \eta+\varphi_{j}\right)$. Hence, with the use of the generating function $e^{i \xi \sin \theta}=\sum_{k=-\infty}^{\infty} J_{k}(\xi) e^{i k \theta}$ of the Bessel functions $J_{k}$, we can expand an input oscillation $e^{i \omega_{0} s}$ into a series of output harmonics $\exp \left[i\left(\omega_{0}+\right.\right.$ $\left.\left.\sum_{j} q_{j} \omega_{j}\right) \eta\right]$. Then, we can approximate the spectrum of the field at the output of a series of $M_{a}$ and $M_{b}$ as the product of the Bessel-function spectra of the individual cells [7]. The condition $\alpha_{j} z_{j} \ll 1$ means that the pulse bandwidths $\gamma_{j} z_{j} \omega_{j}[1,4]$, with $\gamma_{j}=\alpha_{j} \omega_{0} / \omega_{j}$, produced by the individual cells, are small compared to the optical carrier frequency $\omega_{0}$. The same results are also obtained for the case of mixed cells (except that individual cell lengths $z_{j}$ should be replaced by a common length $z$ ). Under the condition of small pulse bandwidths, there is no interference between $\chi_{a}$ and $\chi_{b}$, and therefore, no difference between the mixture and cascades of $M_{a}$ and $M_{b}$ in frequency modulation and pulse compression.

Finally, we demonstrate a numerical example for a realistic system, namely, for a cell containing mixed $\mathrm{H}_{2}$ and $\mathrm{D}_{2}$ molecules. We take $\omega_{a}=587 \mathrm{~cm}^{-1}$ and $\omega_{b}=179$ $\mathrm{cm}^{-1}$ so as to correspond to the rotational transitions of $\mathrm{H}_{2}$ and $\mathrm{D}_{2}$. We use a probe with a carrier frequency $\omega_{0}=20000 \mathrm{~cm}^{-1}$, a pulse length $T=4.5 \mathrm{ps}$, and a peak time $\eta_{p}=0$. We assume the comb depths $\alpha_{a} z=0.587$ and $\alpha_{b} z=0.179$, which correspond to the modulation depths $\gamma_{a} z=\gamma_{b} z=20$. Similar to the results of Ref. [7] for the case of a cascade, a broad spectrum with a large number of sidebands, ranging from $8000 \mathrm{~cm}^{-1}$ to 46000 $\mathrm{cm}^{-1}$, is generated. Unlike the results of Ref. [7], the spectrum in Fig. A(a) is asymmetric. Such asymmetry is due to the deviation of the spectrum from the Besselfunction spectrum, and occurs when the comb depths $\alpha_{j} z$ are not too small compared to unity. The output field in Fig. A(b) is a long train of slightly compressed sections. As has been shown in Ref. [7] for the case of a cascade, single-cycle pulses can be synthesized when the sidebands are phase-corrected by a phase compensator. We illustrate in Fig. A(c) a single-cycle pulse synthesized from the phase-corrected spectrum of Fig. A(a).

In summary, we have studied beating of a probe field with a time-varying susceptibility of a Raman medium. We have derived a general analytical solution and conservation relations for this process. We have shown that the interference between Raman polarizations mav substan-
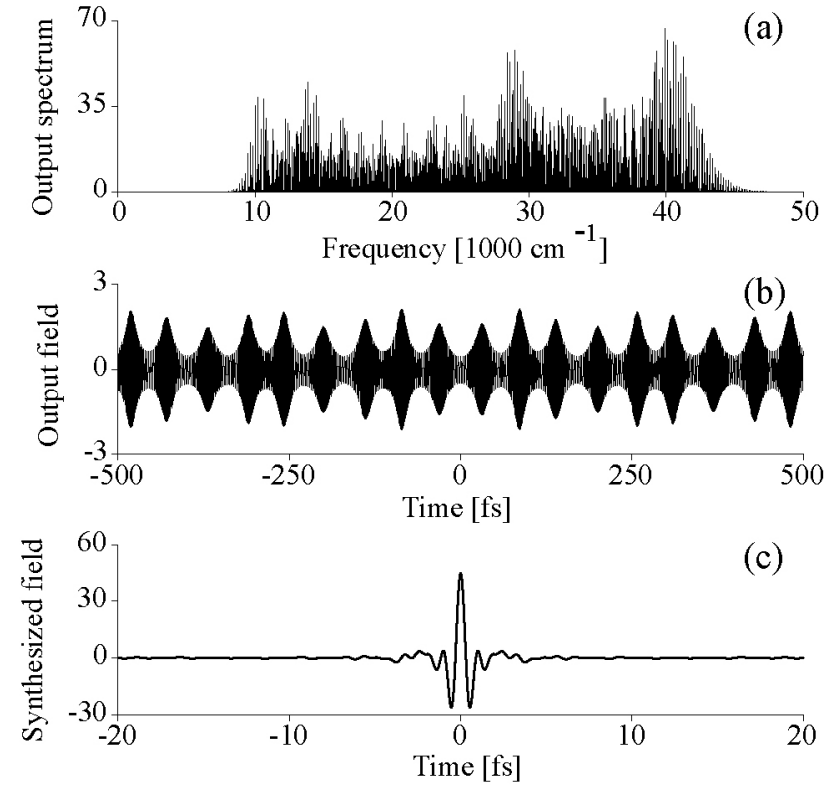

FIG. 4: Spectrum (a), output field (b), and time-domain synthesis by a phase compensator (c) for a cell containing $\mathrm{H}_{2}$ and $\mathrm{D}_{2}$. The parameters for the medium are $\omega_{a}=587 \mathrm{~cm}^{-1}$, $\omega_{b}=179 \mathrm{~cm}^{-1}, \alpha_{a} z=0.587, \alpha_{b} z=0.179$, and $\varphi_{a}=\varphi_{b}=0$. The carrier frequency, pulse length, and peak time of the input field are $\omega_{0}=20000 \mathrm{~cm}^{-1}, T=4.5 \mathrm{ps}$, and $\eta_{p}=0$, respectively. The peak value of the input field is one unit. The synthesized field is obtained when the sidebands are phasecorrected to the same phase of zero.

tially affect frequency modulation and pulse compression. We emphasize that the analysis of this work can be directly applied only when dispersion is negligible. When dispersion becomes substantial, our analytical solution and conservation relations still provide a useful insight, but a numerical simulation of pulse propagation is necessary in order to obtain an exact solution.

We thank A. K. Patnaik for helpful discussions. A. V. S. acknowledges support from the Welch Foundation.
[1] Fam Le Kien, K. Hakuta, and A. V. Sokolov, LANL eprint: quant-ph/0207091 (to be published in Phys. Rev. A).

[2] S. E. Harris and A. V. Sokolov, Phys. Rev. A 55, R4019
(1997); A. V. Sokolov, D. D. Yavuz, and S. E. Harris, Opt. Lett. 24, 557 (1999).

[3] A. V. Sokolov, D. R. Walker, D. D. Yavuz, G. Y. Yin, and S. E. Harris, Phys. Rev. Lett. 85, 562 (2000); A. 
V. Sokolov, D. D. Yavuz, D. R. Walker, G. Y. Yin, and S. E. Harris, Phys. Rev. A 63, 051801(R) (2001); J. Q. Liang, M. Katsuragawa, Fam Le Kien, and K. Hakuta, Phys. Rev. Lett. 85, 2474 (2000); M. Katsuragawa, J. Q. Liang, Fam Le Kien, and K. Hakuta, Phys. Rev. A 65, 025801 (2002).

[4] S. E. Harris and A. V. Sokolov, Phys. Rev. Lett. 81, 2894 (1998); Fam Le Kien, J. Q. Liang, M. Katsuragawa, K. Ohtsuki, K. Hakuta, and A. V. Sokolov, Phys. Rev. A 60, 1562 (1999).

[5] A. V. Sokolov, D. R. Walker, D. D. Yavuz, G. Y. Yin, and S. E. Harris, Phys. Rev. Lett. 87, 033402 (2001).

[6] N. Zhavoronkov and G. Korn, Phys. Rev. Lett. 88,
203901 (2002).

[7] S. E. Harris, D. R. Walker, and D. D. Yavuz, Phys. Rev. A 65, 021801(R) (2002).

[8] V. Kalosha, M. Spanner, J. Herrmann, and M. Ivanov, Phys. Rev. Lett. 88, 103901 (2002).

[9] R. A. Bartels, T. C. Weinacht, N. Wagner, M. Baertschy, Chris H. Greene, M. M. Murnane, and H. C. Kapteyn, Phys. Rev. Lett. 88, 013903 (2002).

[10] V. P. Kalosha and J. Herrmann, Phys. Rev. Lett. 85, 1226 (2000); Fam Le Kien, Nguyen Hong Shon, and K. Hakuta, Phys. Rev. A 64, 051803(R) (2001). 